\title{
Analysis of Peripheral Blood Cells Due to Adults Posthepatitic Cirrhotic Portal Hypertension and Their Postoperative Prognosis
}

\author{
Yunfu Lv*, XiaoYu Han, Xiaoguang Gong, Wenbiao Gu, Chao He, Hongfei Wu, YeJuan Li and Jie Den
}

Department of General Surgery, Hainan Province People's Hospital, Haikou 570311, China

\begin{abstract}
Objectives: To explore the constituent ratio of peripheral blood cells changes in patients with splenomegaly due to posthepatitic cirrhotic portal hypertension and its postoperative prognosis, and to grade the peripheral cytopenias.

Methods: Data of 366 cases of splenomegaly due to posthepatitic cirrhotic portal hypertension were collected and analyzed.

Results: Thirty-six patients (9.8\%) had a normal blood cell count and 330 patients had peripheral cytopenias, in which mono-lineage cytopenias accounted for $30 \%$ (99/330), bi-lineage cytopenias accounted for $35.8 \%(118 / 330)$ and tri-lineage cytopenias accounted for $34.2 \%(113 / 330)$. There were significant statistical differences $(P<0.05)$ in the postoperative prognostic comparison between mono-lineage cytopenias and multi-linage cytopenias. In the 330 cases of cytopenias, the postoperative prognosis demonstrated significant differences between thrombocytopenia, leukopenia and erythropenia $(P<0.05)$. For the most part, according to the severity of thrombocytopenia, cytopenia was graded as mild, medium and severe, and scored as $<2$ points, $2-3$ points and $>3$ points, respectively. The prognosis revealed significant differences between the three grades $(P<0.05)$.
\end{abstract}

Conclusions: Peripheral cytopenias can influence postoperative prognosis. Thrombocytopenia is a major factor influencing postoperative prognosis and grading is of great significance in analyzing the condition of inpatients and guiding their treatment.

Keywords: Adult; Peripheral change; Portal hypertension; Postoperative prognosis

\section{Introduction}

Patients with splenomegaly due to posthepatitic cirrhotic portal hypertension usually experience severe gastrointestinal hemorrhage, hypersplenism and hepatocarcinoma, and should receive surgical operations. Multiple factors influencing the postoperative prognosis exist [1], and peripheral cytopenias are one of these important factors. Peripheral cytopenias indicate that the blood cell count in blood sample from the peripheral vein is lower than normal, i.e., the leukocyte (WBC) count is $<4.0 \times 10^{9} / \mathrm{L}$, the erythrocyte $(\mathrm{RBC})$ count is $<4.0 \times 10^{12} / \mathrm{L}$ or the platelet (PLT) count is $<100 \times 10^{9} / \mathrm{L}$ when first got into the hospital. In this study, data were collected from 366 patients with splenomegaly due to posthepatitic cirrhotic portal hypertension from January 1993 to June 2013, and the constituent ratio of peripheral cytopenias and its influence on the postoperative prognosis were analyzed.

\section{Clinical Data}

\section{General information}

This study included 250 male patients and 116 female patients (a total of 366 patients), and the ratio of males to females was 2.2:1. The patients' ages ranged from 18 to 79 years, with an average of 44 years. All the patients had B hepatic cirrhosis and an enlarged spleen. The average spleen size was $224 \mathrm{~mm} \times 159 \mathrm{~mm} \times 95 \mathrm{~mm}$, as measured by B ultrasound or CT scan. Upper gastrointestinal imaging and gastroscopy revealed that there were medium-to-severe varices in the distal esophagus and gastric fundus. Seventy-four patients (20.2\%) were hospitalized for gastrointestinal hemorrhage, and 248 (67.8\%) patients had previously experienced hemorrhaging. All the patients received a splenectomy after blood transfusion to supplement deficient leukocytes (WBC), erythrocytes (RBC) or platelets (PLT). In addition, 356 patients received extensive devascularization around the cardia (plus a splenorenal vein shunt in 56 patients), 5 received a mesocaval shunt and 5 received a portacaval shunt.

\section{Statistical Analysis}

All data were processed withf SPSS 18.0. Statistical significance was assessed using the $\chi^{2}$-test for univariate analysis or multiple linear regression for multivariate analysis, and $\mathrm{p}<0.05$ was considered significant.

\section{Results}

Among the 366 patients, 36 patients (9.8\%) had normal blood cell counts, while 330 patients $(90.2 \%)$ had peripheral cytopenias, in which mono-lineage cytopenias accounted for 30\% (99/330), bi-lineage cytopenias accounted for $35.8 \%$ (118/330), and tri-lineage cytopenias accounted for $34.2 \%(113 / 330)$. The postoperative prognosis was classified as cured, improved or dead. There were few cases without any changes. In this analysis, cured meant meeting the following criteria: the disappearance of ascites, abdominal distension and hemorrhage,

*Corresponding author: Yunfu Iv, Department of General Surgery, Hainan Province People's Hospital, Haikou 570311,China, Tel: 086898-68723574; E-mail: yunfu_lv@126.com

Received Septemebr 23, 2015; Accepted October 31, 2015; Published November 07, 2015

Citation: Yunfu Lv, Han XY, Gong X, Gu W, He C, et al. (2015) Analysis of Peripheral Blood Cells Due to Adults Posthepatitic Cirrhotic Porta Hypertension and Their Postoperative Prognosis. J Hypertens (Los Angel) 4: 210. doi:10.4172/2167-1095.1000210

Copyright: ( 2015 Yunfu Lv, , et al. This is an open-access article distributed unde the terms of the Creative Commons Attribution License, which permits unrestricted use, distribution, and reproduction in any medium, provided the original author and source are credited. 
Citation: Yunfu Lv, Han XY, Gong X, Gu W, He C, et al. (2015) Analysis of Peripheral Blood Cells Due to Adults Posthepatitic Cirrhotic Portal Hypertension and Their Postoperative Prognosis. J Hypertens (Los Angel) 4: 210. doi:10.4172/2167-1095.1000210

Page 2 of 4

blood cell count increase and recovery, improvement in liver function, no severe postoperative complications, and meeting the criteria for being discharged from the hospital. On the other hand, dead meant that the patients died during hospitalization, or that the patients in critical condition died one week after early discharge from the hospital, as requested by the relatives. All others were considered improved. Comparison of the therapeutic effect between each mono-lineage cytopenia group is shown in Table 1. Comparison of the therapeutic effect between mono-lineage cytopenia and bi-lineage and comparisons of the therapeutic effect between the mono-lineage cytopenia and bi-lineage cytopenias, the mono-lineage cytopenia and multi-lineage cytopenias are shown in Tables 2 and 3, respectively.

The decrease in PLT was the major factor influencing the postoperative prognosis compared with decreases in $\mathrm{WBC}, \mathrm{RBC}$ and $\mathrm{HB}$ (Table 4); in this case, there was significant difference $(\mathrm{P}<0.005)$, while decreases in $\mathrm{WBC}, \mathrm{RBC}$ and $\mathrm{HB}$ showed no significant difference ( $\mathrm{P}>0.05)$.

The 330 cases of cytopenia scores were mainly based on thrombocytopenia combined with erythropenia, as well as clinical experience (leukopenia). Scoring was as follows: PLT $>50<100 \times 10^{9} / \mathrm{L}$ was scored as 1 point, $30-50 \times 10^{9} / \mathrm{L}$ was scored as 2 points, $<30 \times 10^{9} / \mathrm{L}$ was scored as 3 points; RBC $3-4 \times 10^{12} / \mathrm{L}$ was scored as 0 points, and $\mathrm{RBC}<3 \times 10^{12} / \mathrm{L}$ was scored as 1 point; $\mathrm{WBC} 2-4 \times 10^{9} / \mathrm{L}$ was scored as 0 points, and $\mathrm{WBC}<2 \times 10^{9} / \mathrm{L}$ was scored as 1 point. Except for 36 cases with normal blood cell counts and 69 cases with 0 points, the influences of scores on postoperative prognoses in 261 cases are shown in Table 5 (totally 105 cases). There were significant differences between the 3 groups $(\mathrm{P}<0.05)$. Therefore, peripheral cytopenias were graded as mild $(<2)$, medium $(2-3)$ or severe $(>3)$ (Table 6$)$.

\section{Discussion}

In this study, approximately $10 \%$ of patients with splenomegaly due to posthepatitic cirrhotic portal hypertension had normal peripheral blood cell counts, while $90 \%$ had peripheral cytopenia [2], of which $70 \%$ had multi-lineage cytopenias and $30 \%$ had mono-lineage cytopenias. Table 1 shows that only the RBC group demonstrated a significant difference $(\mathrm{P}<0.05)$ in the intra-group comparison among the mono-lineage cytopenia groups. According to Tables 2 and 3 , there were significant differences $(\mathrm{P}<0.05)$ in the therapeutic effects between mono-lineage cytopenias and multi-lineage cytopenias, indicating that the more severe the cytopenia, the worse the therapeutic results appeared to be.

For the multi-lineage cytopenias, a multiple linear regression analysis was applied, and results revealed that thrombocytopenia was the major factor $(\mathrm{P}<0.005)$ influencing the therapeutic effect, while leukopenia, erythropenia and decreased hemoglobin showed no statistical significance, and should not be considered. Erythropenia showed significant differences in the intra-group comparison of monolineage cytopenias, but no difference compared to other mono-lineage cytopenia groups. This was possibly due to the small sample size in the mono-lineage cytopenia groups. Leukopenia showed no significant difference in the univariate analysis or the multivariate analysis, and had no influence on the therapeutic results. For example, 2 patients recovered and were discharged from the hospital though their leukocyte count was lower than $1 \times 10^{9} / \mathrm{L}$; this may have been because they had no serious postoperative infection. Theoretically, anemia is related to the prognosis, but in this research it showed no statistical significance in the univariate analysis or the multivariate analysis; the reason for

\begin{tabular}{|c|c|c|c|c|c|c|}
\hline \multirow[t]{2}{*}{ Group } & \multirow[t]{2}{*}{ Grade } & \multirow[t]{2}{*}{ Case number } & \multicolumn{3}{|c|}{ Therapeutic effect } & \multirow[t]{2}{*}{$\mathrm{X}^{2}, \mathrm{P}$ value } \\
\hline & & & Cured (\%) & Improved (\%) & Dead (\%) & \\
\hline WBC & $<2$ & 1 & $1(100)$ & 0 & 0 & $x^{2}=1.478, P=0.478$ \\
\hline$(\times 109 / \mathrm{L}$ & $3-F e b$ & 10 & $6(60)$ & $4(40)$ & 0 & \\
\hline $\mathrm{N}=14$ ) & 4-Mar & 3 & $1(33.3)$ & $2(66.7)$ & 0 & \\
\hline RBC & $<2$ & 4 & $3(75)$ & 0 & $1(25)$ & $x^{2}=10.908$ \\
\hline$(\times 1012 / \mathrm{L}$ & $3-F e b$ & 20 & $16(80)$ & $2(10)$ & $2(10)$ & $P=0.028<0.05$ \\
\hline $\mathrm{N}=58)$ & 4-Mar & 34 & $16(47.1)$ & $16(47.1)$ & $2(5.8)$ & \\
\hline PLT & $<30$ & 3 & $1(33.3)$ & $2(66.7)$ & 0 & $X^{2}=2.220, P=0.695$ \\
\hline$(\times 109 / \mathrm{L}$ & $30-50$ & 1 & $1(100)$ & 0 & 0 & \\
\hline $\mathrm{N}=27$ ) & $50-100$ & 23 & $15(65.2)$ & $7(30.4)$ & $1(4.4)$ & \\
\hline HB (hemoglobin) & $<30$ & 78 & $32(41)$ & $39(50)$ & $7(9)$ & $x^{2}=4.236, P=0.375$ \\
\hline$(\times \mathrm{g} / \mathrm{L}$ & $30-70$ & 52 & $28(53.8)$ & $20(38.5)$ & $4(7.7)$ & \\
\hline$N=366)$ & $>70$ & 236 & $122(51.7)$ & $89(37.7)$ & $25(10.6)$ & \\
\hline
\end{tabular}

Note: Among the four mono-lineage peripheral cytopenia groups, only the RBC group demonstrated a significant difference in intra-group comparison ( $p<0.05$ ); comparison among the four groups revealed no significant difference $(P>0.05)$

Table 1: Comparison of the therapeutic effect between each mono-lineage peripheral cytopenia group.

\begin{tabular}{|l|c|c|c|c|}
\hline \multicolumn{1}{|c|}{ Item } & Total case number & \multicolumn{3}{|c|}{ Therapeutic effect } \\
\hline Mono-lineage cytopenia & \multirow{2}{*}{ X } & Cured (\%) & Improved (\%) & Dead (\%) \\
\cline { 2 - 5 } & & $60(60.6 \%)$ & $33(33.3 \%)$ & $6(6.1 \%)$ \\
\hline Bi-lineage cytopenia & 118 & $51(43.2 \%)$ & $51(43.2 \%)$ & $16(13.6 \%)$ \\
\hline
\end{tabular}

Table 2: Comparison of the therapeutic effect between the mono-lineage cytopenia and bi-lineage cytopenia.

\begin{tabular}{|c|c|c|c|c|}
\hline \multirow{2}{*}{ Item } & Total case number & \multicolumn{3}{|c|}{ Therapeutic effect } \\
\hline \multirow{2}{*}{ Mono-lineage cytopenia } & & Cured (\%) & Improved (\%) & Dead (\%) \\
\cline { 2 - 5 } & 99 & $60(60.6)$ & $33(33.3)$ & $6(6.1)$ \\
\hline Multi-lineage cytopenia & 231 & $102(44.2)$ & $102(44.2)$ & $27(11.6)$ \\
\hline
\end{tabular}

Table 3: Comparison of the therapeutic effect between the mono-lineage cytopenia and tri-lineage cytopenia. 


\begin{tabular}{|c|c|c|}
\hline Item & T value & P value \\
\hline PLT & 2.827 & 0.005 \\
\hline RBC & -0.439 & 0.661 \\
\hline WBC & 1.516 & 0.13 \\
\hline HB & 0.628 & 0.531 \\
\hline Constant & 1.395 & 0 \\
\hline
\end{tabular}

Note: Regression equation $\hat{Y}=1.395+0.151$ PLT

Table 4: Multiple linear regression analysis of blood cells in 366 cases

\begin{tabular}{|c|c|c|c|c|c|}
\hline \multirow{2}{*}{ Item } & $\begin{array}{c}\text { Total case } \\
\text { number }\end{array}$ & \multicolumn{3}{|c|}{ Therapeutic effect } & \multirow{2}{*}{ X2, P value } \\
\hline 1 point & 136 & Cured (\%) & Improved (\%) & Death (\%) & \\
\cline { 3 - 6 } & $80(58.8 \%)$ & $43(31.6 \%)$ & $13(9.6 \%)$ & $\mathrm{X} 2=10.163$ \\
\hline 2-3 points & 95 & $41(43.2 \%)$ & $44(46.3 \%)$ & $10(10.5 \%)$ & $\mathrm{P}=0.034$ \\
\hline 4-5 points & 30 & $10(33.3 \%)$ & $15(50 \%)$ & $5(16.7 \%)$ & \\
\hline
\end{tabular}

Table 5: Comparison of the influence of different scores on the therapeutic effect.

\begin{tabular}{|c|c|c|c|}
\hline Item & Mild & Medium & Severe \\
\hline PLT & $>50$ & $30-50$ & $<30$ \\
\hline (Score) & 1 & 2 & 3 \\
\hline RBC & $>3$ & $3-F e b$ & $<2$ \\
\hline$($ Score $))$ & 0 & 1 & 1 \\
\hline WBC & $>3$ & $3-F e b$ & $<2$ \\
\hline (Score) & 0 & 0 & 1 \\
\hline Total score & $<2$ & $3-F e b$ & $>3$ \\
\hline
\end{tabular}

Table 6: Grading of peripheral cytopenias (hypersplenism).

this may have been because the blood transfusions before and during operation had a favorable effect on the blood condition. Although thrombocytopenia had no statistical significance in the univariate analysis, in the multiple linear regression analysis it was indicated to be the most important influential factor with the increase in case load.

Thrombocytopenia is a significant and common complication in posthepatitic cirrhotic portal hypertension $[3,4]$; it is related to not only retention of blood cell in the spleen, blood cell aggregation and enhanced phagocytosis of macrophages [5], but also HBV infection, and compensation and regulation of marrow [6]. Djordevic et al. [7,8]. Proposed that extreme thrombocytopenia was life-threatening. A PLT count of $<30 \times 10^{9} / \mathrm{L}$ can cause variceal hemorrhaging in the distal esophagus and gastric fundus, and intraoperative and postoperative massive wound hemorrhaging, which can be life-threatening. Therefore, PLT transfusions should be performed before an operation to increase the PLT count to $50 \times 10^{9} / \mathrm{L}$ to ensure the safety of the patient. Cui et al. [9] reported that PLT transfusions combined with plasma fibrinogen transfusions led to better results. In some cases, after transfusion of 1224 units of PLT, the PLT count did not increase obviously, or decreased to the previous lowest count after 1-2 days. These types of patients are suitable for splenectomy [10]. Mastuura et al. [11] suggested that the excessive postoperative PLT count was also a life-threatening factor, so the condition of the patient should be closely monitored [12,13] when there is excessive platelet count, appropriate treatment should be administrated immediately.

In 1907, Chauffard proposed the term 'hypersplenism' for the first time [14]. After further research, in 1949, Doan [15] proposed the criteria for hypersplenism: 1. enlarged spleen 2. mono-lineage or multi-lineage cytopenias 3. normal or proliferative bone marrow 4 . disappearance of the pathological changes in the blood components after splenectomy. While these four criteria are indispensable for the diagnosis of hypersplenism, peripheral cytopenia and an increase and recovery in blood cell count after a splenectomy are the major criteria for assessing hypersplenism due to cirrhotic portal hypertension. This is because splenomegaly in itself is a necessary criterion for the cirrhotic portal hypertension.

Grading hypersplenism is very difficult as there are mono-lineage cytopenias, bilineage cytopenia or pancytopenia, and even in the pancytopenia; thus, it is difficult to meet the criteria for grading. On the other hand, there are many causes for cytopenias in cirrhotic portal hypertension; therefore, it is very difficult to identify which type of cytopenia is caused by hypersplenism preoperatively. Thus, only cytopenias can be graded. In the present study, the cases were scored and graded based on the accumulated scores. The scoring criteria used in this study were: 1 . Analytical results of multiple linear regression: F value obtained from multiple linear regression equation was 7.993 $(\mathrm{P}<0.005)$, indicating that multiple linear regression was applicable. The equation $\hat{Y}=1.395+0.151$ PLT indicated that thrombocytopenia was the major influential factor for postoperative prognosis. Therefore, according to the severity of the thrombocytopenia, 1 to 3 points was scored. 2. Intra-group comparison of erythropenia showed a significant difference $(\mathrm{P}<0.05)$, so an $\mathrm{RBC}$ count $\leq 3 \times 10^{12} / \mathrm{L}$ was scored as 1 point. 3. According to clinical experience, leukopenia can cause severe infection and lead to undesirable effects. A WBC of count $\leq 2 \times 10^{9} / \mathrm{L}$ was scored as 1 point, though leukopenia showed no statistical significance in either the univariate analysis or multivariate analysis. A total score of $<2$ points indicated mild cytopenia, 2-3 points indicated medium cytopenia and $>3$ points indicated severe cytopenia. If cytopenias are caused by hypersplenism, this grading standard could also be used for grading hypersplenism or as a reference [16].

Cytopenia grading could facilitate clinical practice in various aspects, including assessing the disease condition, representation and academic communication, communication with patients and their relatives to resolve or avoid medical disputes, choosing a suitable treatment plan (for example, splenectomy is suitable for severe cytopenia or hypersplenism) and taking preventive methods before an operation $[16,17]$ to increase the curative rate.

\section{Acknowledgement}

Special projects Funded by the Special Fund for Scientific and Technological Cooperation of Hainan, China (Project No.: KJHZ2015-28)

\section{References}

1. Zhen Y, Fa-zhu Q (2003) A study on prognosis of portal hypertension. J Abdom Sur 16: $68-70$.

2. Yun-fu L, Xiao-guang G, Xin-qiu L (2008) Peripheral blood cytopenia in patients with portal hypertension complicated with splenomegaly. Clin J Dig Surg 7: 281-283.

3. Karasu Z, Gurakar A, Kerwin B (2001) Effect of transjuqular intrahepatic portosystemic shunt on thrombocytopenia associated with cirrhosis. Dig Dis Sci 46: 449-456.

4. Bashour FN, Teran JC, Mullen KD (2000) Prevalence of Peripheral Blood Cytopenias Hypersplenism in Patients With Nonalcoholic Liver Disease. Am J Gastroeteroly 95: 2937-2939

5. Yan F, Li W, Chen JT, Zeng YM, Guo YW, et al. (2006) cDNA microarray-based screening of differentially expressed genes in macrophages in the spleen of patients with portal hypertension and hypersplenism. Nan Fang Yi Ke Da Xue Xue Bao 26: 1548-1551.

6. Yun-fu L, Xin-qiu L, Xian-he X (2009) Portal Hypertension Splenomegaly is not Always Associated with Hematocytopenia. J US-China Med Sci 6: 28-30.

7. DjordjeviÄ J, Svorcan P, VriniÄ D, DapceviÄ B (2010) Splenomegaly and thrombocytopenia in patients with liver cirrhosi. Vojnosanit Pregl 67: 166-169.

8. Zhang JY, Wang Y, Zhang J, Ding SG, Zhou LY, et al. (2010) [Risk factors associated with failure from endoscopic therapy in acute non-variceal upper 
Citation: Yunfu Lv, Han XY, Gong X, Gu W, He C, et al. (2015) Analysis of Peripheral Blood Cells Due to Adults Posthepatitic Cirrhotic Portal Hypertension and Their Postoperative Prognosis. J Hypertens (Los Angel) 4: 210. doi:10.4172/2167-1095.1000210

Page 4 of 4

gastrointestinal bleeding]. Beijing Da Xue Xue Bao 42: 703-707.

9. Cui Y, Hei F, Long C, Feng Z, Zhao J, et al. (2009) Perioperative monitoring of thromboelastograph on hemostasis and therapy for cyanotic infants undergoing complex cardiac surgery. Artif Organs 33: 909-914.

10. Yun-fu LV, Xin-qiu LI, Wei-wei Huang (2007) Peripheral blood cytopenia in patients with hypersplenism due to portal hypertension. Clin J Gen Surg 22 97-102.

11. Matsuura T, Hayashida M, Saeki I, Taguchi T (2010) The risk factors of persistent thrombocytopenia and splenomegaly after liver transplantation. Pediatr Surg Int 26: 1007-1010.

12. Johansson PI, Stensballe J (2010) Hemostatic resuscitation for massive bleeding: the paradigm of plasma and platelets--a review of the current literature. Transfusion 50: 701-710

13. Shontz R, Karuparthy V, Temple R, Brennan TJ (2009) Prevalence and risk factors predisposing to coagulopathy in patients receiving epidural analgesia for hepatic surgery. Reg Anesth Pain Med 34: 308-311.

14. Fadi NB, J Carlos T, Kevin D (2000) Prevalence of Peripheral Blood Cytopenias Hypersplenism?in Patients With Nonalcoholic Liver Disease. Am J Gastroeterology 95: 2937-2939.

15. Doan CA (1949) Hypersplenism. Bull N Y Acad Med 25: 625-650.

16. Lu YF, Li XQ, Han XY, Gong XG, Chang SW (2013) Peripheral blood cell variations in cirrhotic portal hypertension patients with hypersplenism. Asian Pac J Trop Med 6: 663-666.

17. Shaz BH, Dente CJ, Nicholas J, MacLeod JB, Young AN, et al. (2010) Increased number of coagulation products in relationship to red blood cell products transfused improves mortality in trauma patients. Transfusion 50: 493-500. 